\title{
LAPS and SPIM imaging using ITO coated glass as the substrate material
}

\author{
De-Wen Zhang ${ }^{\dagger \dagger}$, Fan $\mathrm{Wu}^{\dagger}$, Steffi Krause ${ }^{\dagger *}$ \\ s.krause@qmul.ac.uk \\ ${ }^{\dagger}$ School of Engineering and Materials Science, Queen Mary University of London, Mile End Road, \\ London, E1 4NS, UK \\ ${ }^{\ddagger}$ Institute of Materials, China Academy of Engineering Physics, Jiangyou, 621908, Sichuan, P.R. \\ China
}

\begin{abstract}
Light-addressable potentiometric sensors (LAPS) and scanning photo-induced impedance microscopy (SPIM) use photocurrent measurements for spatiotemporal imaging of ion concentrations, electrical potentials and impedance. In this work, ITO coated glass was confirmed to produce photocurrents at anodic potentials with $405 \mathrm{~nm}$ diode laser illumination. Therefore, it was developed as a low cost and robust substrate material for LAPS and SPIM imaging compared to traditional expensive ultra-thin Si substrates. ITO showed good ac photocurrent and $\mathrm{pH}$ response without surface modification and insulator. Local photocurrents were produced by scanning a focused laser beam across the sample, which proved the light addressability of ITO coated glass. With a high-impedance PMMA dot deposited onto the ITO as a model system, a lateral resolution of about $2.3 \mu \mathrm{m}$ was achieved.
\end{abstract}

\section{Introduction}

Light-addressable potentiometric sensors (LAPS) and scanning photo-induced impedance microscopy (SPIM) are electrochemical imaging techniques based on photocurrent measurements at electrolyteinsulator-semiconductor (EIS) field-effect capacitors ${ }^{1,2}$. By generating photocurrents locally with a focused light-source, they provide information such as surface potential, ion concentrations and electrical impedance with good lateral resolution without the need for a probe.

Bulk silicon was the first and most commonly used semiconductor substrate in LAPS and SPIM ${ }^{3}$. Sensor substrates based on thin silicon ${ }^{4,5}$, porous silicon ${ }^{6}$, and amorphous silicon ${ }^{7-10}$, silicon on insulator $(\mathrm{SOI})^{11}$ and ultra-thin silicon on sapphire $(\mathrm{SOS})^{12,13}$ were developed to improve the photocurrent response and the resolution. Other semiconductor materials such as $\mathrm{GaAs}^{14}, \mathrm{GaN}^{15}$, and $\mathrm{TiO}_{2}{ }^{16}$ were also investigated as alternative sensor materials for LAPS and light-addressable electrodes. 
Theoretical analysis and LAPS device simulation have been developed continuously alongside experimental techniques to improve the lateral resolution ${ }^{11,17-30}$. The spatial resolution in LAPS is limited by the diffusion of minority charge carriers out of the illuminated region, which depends on the size of illuminated area and the average effective diffusion length of minority charge carriers. Good lateral resolution could be achieved by using materials with a small diffusion length $\mathrm{L}_{\mathrm{p}}$ of minority charge carriers such as GaAs $\left(\mathrm{L}_{\mathrm{p}} \sim 3 \mu \mathrm{m}\right)^{14}$ and amorphous $\mathrm{Si}\left(\mathrm{L}_{\mathrm{p}} \sim 100 \mathrm{~nm}\right)^{7-9,31}$ or by using thin films of single crystalline silicon on sapphire (SOS) (effective diffusion length $570 \mathrm{~nm}$ for $1 \mu \mathrm{m}$ thick silicon) ${ }^{11}$.

Besides the average effective diffusion length, the resolution could be improved by the quality of the optical focus. A two-photon effect in silicon produced by a femtosecond laser with energy smaller than the bandgap of silicon was applied to LAPS and SPIM imaging ${ }^{11}$. Charge carriers were only generated in the focus but not throughout the bulk of the semiconductor, and the effects of stray light and reflections at edges were eliminated. Based on a $0.5 \mu \mathrm{m}$ thick boron doped SOS substrate and a two-photon effect in silicon, submicrometer resolution of $0.8 \mu \mathrm{m}$ was obtained ${ }^{12}$. This measurement system was used for imaging the impedance of microcapsules (diameter $\sim 5 \mu \mathrm{m}$ ) with high resolution $^{32}$. Self-assembled organic monolayers patterned by microcontact printing $(\mu \mathrm{CP})$ were visualized by LAPS and SPIM through local changes in the potential and the impedance ${ }^{13,33}$. The disadvantage of SOS is the high cost of the substrate, which has limited the widespread use of SOS in LAPS.

In this work, indium tin oxide (ITO) coated glass was investigated as a new substrate material for LAPS and SPIM imaging. ITO is a heavily doped n-type semiconductor with a large bandgap of around $3.5 \mathrm{eV}$ to $4.3 \mathrm{eV}^{34}$. It is widely used as transparent conducting oxide because of its electrical conductivity, optical transparency and the ease to be deposited as films. In this work, a photocurrent of blank ITO could be excited by the UV part of a $405 \mathrm{~nm}$ diode laser that has been commonly used as a light source in LAPS. The mature ITO coated glass is cheap, robust, stable and easy to be modified, making it a suitable substrate for LAPS and SPIM imaging. It could significantly expand the application of LAPS imaging for general use. The photocurrent response, the $\mathrm{pH}$ response, LAPS and SPIM imaging and its lateral resolution using ITO coated glass without any modification were investigated in this work.

\section{Experimental Section}

\section{Materials}

Indium tin oxide (ITO) coated glass $\left(50 \Omega / \mathrm{cm}^{2}\right)$ was purchased from Diamond Coatings Limited, UK. All chemicals were purchased form Sigma-Aldrich. All solutions were prepared using ultrapure water $(18.2 \mathrm{M} \Omega \mathrm{cm})$ from a Milli-Q water purification system (Millipore, USA). 


\section{Preparation of ITO coated glass}

The ITO coated glass was cut into $1 \mathrm{~cm} \times 1 \mathrm{~cm}$ pieces. They were cleaned by ultrasonication for 15 min with acetone, isopropanol, and ultrapure water. After drying with nitrogen, the ITO coated glass was kept at room temperature before use.

\section{Photocurrent measurements}

Linear sweep voltammetry (LSV) was carried out in $10 \mathrm{mM} \mathrm{pH} 7.4$ phosphate buffer with $0.1 \mathrm{M} \mathrm{KCl}$ using an Autolab PGSTAT30/FRA2 (Windsor Scientific Ltd, UK) using a platinum electrode and a $\mathrm{Ag} / \mathrm{AgCl}$ electrode as the counter and reference electrodes, respectively. The scan rate was $5 \mathrm{mV} / \mathrm{s}$. A diode laser ( $\lambda=405 \mathrm{~nm}$, max $500 \mathrm{~mW}$ ) was used as the light source.

\section{LAPS measurements}

The LAPS setup is shown in Figure 1. A focused diode laser LD1539 (Laser 2000, $\lambda=405 \mathrm{~nm}$, max $50 \mathrm{~mW}$, focused spot diameter $\sim 1 \mu \mathrm{m}$ ) was used for charge carrier generation. The laser intensity was modulated at a frequency of $10 \mathrm{~Hz}$ to generate ac photocurrents. The positon of the sample holder was adjusted by an M-VP-25XL XYZ positioning system with a $50 \mathrm{~nm}$ motion sensitivity on all axes (Newport, UK). AC photocurrents were measured by an EG\&G 7260 lock-in amplifier using a platinum electrode and a $\mathrm{Ag} / \mathrm{AgCl}$ electrode as the counter and reference electrodes, respectively.

\section{Results and Discussion}

\section{The photocurrent response of ITO coated glass}

The blank ITO coated glass showed an obvious photocurrent using LSV at anodic potentials with chopped $405 \mathrm{~nm}$ diode laser illumination (Figure 2). Based on a bandgap of $4.3 \mathrm{eV}$ to $3.5 \mathrm{eV}$, charge carriers in ITO should be excited at wavelengths below $288 \mathrm{~nm}$ to $354 \mathrm{~nm}$, which indicated that charge carrier excitation was caused by the UV part of the wavelength distribution of the diode laser. This was also confirmed by a UV-Vis spectrum of the ITO coated glass (Figure S1) that shows that the material is transparent to visible light and only absorbs light at wavelengths $<430 \mathrm{~nm}$. Compared to a negligible background dark current, the photocurrent under illumination increased with the applied potential. An impedance spectrum of the ITO in PBS (Figure S2) was dominated by a double layer capacitance of $13.2 \mu \mathrm{F} / \mathrm{cm}^{2}$ over a large range of frequencies demonstrating the blocking nature of the ITO-solution interface in the dark. This provided the basis for using ITO coated glass as a substrate for LAPS and SPIM.

The $\mathrm{pH}$ response of ITO coated glass 
LAPS has been developed for the detection of $\mathrm{pH}$, ions, redox pairs and enzymatic reactions ${ }^{2}$. As the photocurrent-voltage $(I-V)$ curve of LAPS responds to the surface potential, the $\mathrm{pH}$ could be monitored by a potential shift with a $\mathrm{pH}$-sensitive layer, which has become a standard test. The $I-V$ responses of ITO coated glass were measured in a series of $\mathrm{pH}$ phosphate buffer solutions ( $\mathrm{pH} 4-9$ ) with $0.1 \mathrm{M} \mathrm{KCl}$ using the LAPS setup shown in Figure 1. The ITO coated glass sensor showed an average $\mathrm{pH}$ sensitivity of $70 \mathrm{mV} / \mathrm{pH}$ at a constant photocurrent of $10 \mathrm{nA}$ (Figure 3) making this a promising $\mathrm{pH}$ imaging sensor that could be adapted for other biochemical analysis with LAPS. After measuring $I$ - $V$ curves in buffer solutions from $\mathrm{pH} 4$ to 9 in sequence, the $I$ - $V$ curve returned to its initial positon when the $\mathrm{pH}$ was changed back to 4 , showing good reversibility and stability of ITO coated glass for $\mathrm{pH}$ sensing. In contrast to traditional LAPS measurements where the semiconductor substrate is insulated, the ac photocurrent measured with blank ITO originates from the anodic photocurrent shown in Figure 2, which is affected by the $\mathrm{pH}$ dependent kinetics of the anodic oxidation process.

\section{The LAPS and SPIM imaging of ITO coated glass}

Figure 4 (A) shows the LAPS I- $V$ characteristics of ITO coated glass with an unfocused laser source modulated at $10 \mathrm{~Hz}$ in $\mathrm{pH} 7.4$ phosphate buffer. The ac photocurrent increased with the bias voltage and reached a plateau at about $2.3 \mathrm{~V}$. At voltages greater than $2.5 \mathrm{~V}$, the photocurrent dropped to zero because of the production of oxygen bubbles on the ITO surface at high positive potentials. In the following experiments, $2 \mathrm{~V}$ was, therefore, chosen as the general measurement voltage.

To investigate the effect of the modulation frequency on the photocurrent, both, the photocurrent and dark current at $2 \mathrm{~V}$, were measured from $10 \mathrm{~Hz}$ to $10000 \mathrm{~Hz}$. As shown in Figure 4 (B), the ac photocurrent decreased with increasing frequency while the dark current increased. The signal to noise ratio of the photocurrent decreased with increasing frequency. At frequencies greater than $10000 \mathrm{~Hz}$ the photocurrent could not be distinguished from the background dark current. $10 \mathrm{~Hz}$ was chosen as the measurement frequency in this work. To increase the measurement speed, higher frequencies up to $1000 \mathrm{~Hz}$ could also be used, albeit at the cost of a decreased signal to noise ratio and, therefore, somewhat reduced LAPS and SPIM imaging quality.

To investigate the light-addressability of ITO coated glass, the blank ITO sample was used for photocurrent imaging. The modulated laser beam was focused through the glass substrate onto the ITO layer. A scan of the photocurrent with the distance of the sample from the microscope objective (z axis) was used to find the laser beam focus (Figure 5 (B)). The photocurrent reached a minimum in focus as the amount of photo-generated carriers decreased with the decrease of the illuminated area when focusing. The focused spot diameter was about $1 \mu \mathrm{m}$. The LAPS $I-V$ curve in focus showed similar but lower photocurrent response compared to the unfocused laser source (Figure $5(\mathrm{C})$ ). A 
typical $100 \times 100$ pixel photocurrent image of a circular area of blank ITO sealed with a rubber Oring is shown in Figure $5(\mathrm{~A})$.

To characterize the lateral resolution, a poly (methyl methacrylate) (PMMA) dot was deposited onto the ITO coated glass surface. Figure 6 (A) shows the SPIM area scan of the PMMA dot on the ITO coated glass surface measured at a bias voltage of $2 \mathrm{~V}$ and a modulation frequency of $10 \mathrm{~Hz}$ with a focused laser beam. The polymer dot is clearly visible as a reduction in photocurrent in the coated area. In the polymer dot covered area, the photocurrent decreased to nearly zero because of the high impedance of the PMMA dot. For lateral resolution measurements, a photocurrent line scan across the edge of the polymer film was carried out with the focused laser beam and $1 \mu \mathrm{m}$ step size (Figure 6 (B)). The full width at half maximum (FWHM) value of the first derivative of the edge response is a typical indicator of the lateral resolution ${ }^{22}$. As shown in Figure $6(\mathrm{C})$, the FWHM lateral resolution was about $2.3 \mu \mathrm{m}$, which is comparable to ultra-thin silicon on sapphire illuminated with a $405 \mathrm{~nm}$ blue laser, which showed a resolution of $1.5 \mu \mathrm{m}^{12}$. The good lateral resolution can be attributed to the small thickness of the ITO coating in the order of hundreds of nanometers resulting in a small effective diffusion length of minority charge carriers and the high quality focus of the laser beam.

\section{Conclusions}

ITO coated glass was tested as a substrate material for LAPS and SPIM imaging. It showed an obvious photocurrent when illuminated with a $405 \mathrm{~nm}$ diode laser. The unmodified ITO coated glass surface showed a repeatable $\mathrm{pH}$ response in LAPS measurements. Local photocurrents were produced by scanning a focused laser beam across the sample for LAPS and SPIM imaging. Good lateral resolution of $2.3 \mu \mathrm{m}$ was obtained using a PMMA dot deposited on ITO as the model system. The mature, commercial ITO coating technology reduces the cost compared to previously used, expensive semiconductor substrates. The ITO coated glass does not need an insulating layer and an ohmic contact as ITO has good electrical conductivity, which further simplifies the application of ITO coated glass. This simple, low-cost and robust substrate material will make the LAPS and SPIM imaging technology attractive for widespread use for applications that require the measurement of surface potential changes to monitor ion concentrations, the surface attachment of charged molecules or materials, extracellular potentials, and cell surface charges, and local impedance.

\section{Acknowledgements}


The authors are grateful to the EU for providing Marie Skłodowska-Curie Individual Fellowships (H2020-MSCA-IF-2014-660489) and to the China Scholarship Council for providing a PhD studentship to F.W.

\section{Supporting Information}

- UV-Vis spectrum of ITO coated glass

- Impedance spectrum of ITO coated glass in PBS at the open circuit potential in the dark

\section{References}

(1) Yoshinobu, T.; Miyamoto, K.-i.; Werner, C. F.; Poghossian, A.; Wagner, T.; Schöning, M. J. Annual Review of Analytical Chemistry 2017, 10, 19.11-19.22.

(2) Wu, F.; Campos, I.; Zhang, D.-W.; Krause, S. Proceedings of the Royal Society A: Mathematical, Physical and Engineering Science 2017, 473.

(3) Hafeman, D.; Parce, J.; McConnell, H. Science 1988, 240, 1182-1185.

(4) Nakao, M.; Inoue, S.; Yoshinobu, T.; Iwasaki, H. Sensors Actuators B: Chem. 1996, 34, 234-239.

(5) Ito, Y. Sensors Actuators B: Chem. 1998, 52, 107-111.

(6) Yoshinobu, T.; Ecken, H.; Poghossian, A.; Lüth, H.; Iwasaki, H.; Schöning, M. J. Sensors Actuators B: Chem. 2001, 76, 388-392.

(7) Moritz, W.; Yoshinobu, T.; Finger, F.; Krause, S.; Martin-Fernandez, M.; Schöning, M. J. Sensors Actuators B: Chem. 2004, 103, 436-441.

(8) Zhou, Y.; Chen, L.; Krause, S.; Chazalviel, J.-N. Anal. Chem. 2007, 79, 6208-6214.

(9) Zhou, Y.; Jiang, S.; Krause, S.; Chazalviel, J.-N. Anal. Chem. 2007, 79, 8974-8978.

(10) Yoshinobu, T.; Schoning, M. J.; Finger, F.; Moritz, W.; Iwasaki, H. Sensors 2004, 4, 163-169.

(11) Krause, S.; Moritz, W.; Talabani, H.; Xu, M.; Sabot, A.; Ensell, G. Electrochim. Acta 2006, 51, 1423-1430.

(12) Chen, L.; Zhou, Y.; Jiang, S.; Kunze, J.; Schmuki, P.; Krause, S. Electrochem. Commun. 2010, $12,758-760$.

(13) Wang, J.; Zhou, Y.; Watkinson, M.; Gautrot, J.; Krause, S. Sensors Actuators B: Chem. 2015, 209, 230-236.

(14) Moritz, W.; Gerhardt, I.; Roden, D.; Xu, M.; Krause, S. Fresenius J. Anal. Chem. 2000, 367, 329-333.

(15) Das, A.; Das, A.; Chang, L. B.; Lai, C. S.; Lin, R. M.; Chu, F. C.; Lin, Y. H.; Chow, L.; Jeng, M. J. Applied Physics Express 2013, 6 .

(16) Suzurikawa, J.; Nakao, M.; Jimbo, Y.; Kanzaki, R.; Takahashi, H. Sensors Actuators B: Chem. 2014, 192, 393-398.

(17) Sartore, M.; Adami, M.; Nicolini, C. Biosens. Bioelectron. 1992, 7, 57-64.

(18) Massobrio, G.; Martinoia, S.; Grattarola, M. Sensors Actuators B: Chem. 1992, 7, 484-487.

(19) Sartore, M.; Adami, M.; Nicolini, C.; Bousse, L.; Mostarshed, S.; Hafeman, D. Sensors and Actuators A: Physical 1992, 32, 431-436.

(20) Adami, M.; Sartore, M.; Baldini, E.; Rossi, A.; Nicolini, C. Sensors Actuators B: Chem. 1992, 9 , $25-31$.

(21) Parak, W. J.; Hofmann, U. G.; Gaub, H. E.; Owicki, J. C. Sensors and Actuators A: Physical 1997, 63, 47-57.

(22) George, M.; Parak, W. J.; Gerhardt, I.; Moritz, W.; Kaesen, F.; Geiger, H.; Eisele, I.; Gaub, H. E. Sensors and Actuators A: Physical 2000, 86, 187-196. 
(23) Zhang, Q.; Wang, P.; Parak, W. J.; George, M.; Zhang, G. Sensors Actuators B: Chem. 2001, 73, 152-156.

(24) Zhang, Q. Sensors Actuators B: Chem. 2005, 105, 304-311.

(25) Gasparyan, F. V. Journal of Contemporary Physics (Armenian Academy of Sciences) 2010, 45, 228-237.

(26) Werner, C. F.; Wagner, T.; Yoshinobu, T.; Keusgen, M.; Schöning, M. J. physica status solidi (a) 2013, 210, 884-891.

(27) Guo, Y. Y.; Miyamoto, K.; Wagner, T.; Schoning, M. J.; Yoshinobu, T. Physica Status Solidi aApplications and Materials Science 2014, 211, 1467-1472.

(28) Guo, Y.; Seki, K.; Miyamoto, K.-i.; Wagner, T.; Schöning, M. J.; Yoshinobu, T. Applied Physics Express 2014, 7, 067301.

(29) Guo, Y.; Miyamoto, K.-i.; Wagner, T.; Schöning, M. J.; Yoshinobu, T. Sensors Actuators B: Chem. 2014, 204, 659-665.

(30) Litvinenko, S. V.; Kozinetz, A. V.; Skryshevsky, V. A. Sensors and Actuators A: Physical 2015, 224, 30-35.

(31) Das, A.; Lin, Y.-H.; Lai, C.-S. Sensors Actuators B: Chem. 2014, 190, 664-672.

(32) Wang, J.; Campos, I.; Wu, F.; Zhu, J.; Sukhorukov, G. B.; Palma, M.; Watkinson, M.; Krause, S. Electrochim. Acta 2016, 208, 39-46.

(33) Wang, J.; Wu, F.; Watkinson, M.; Zhu, J.; Krause, S. Langmuir 2015, 31, 9646-9654.

(34) Du, J.; Chen, X.-1.; Liu, C.-c.; Ni, J.; Hou, G.-f.; Zhao, Y.; Zhang, X.-d. Appl. Phys. A 2014, 117, 815-822. 


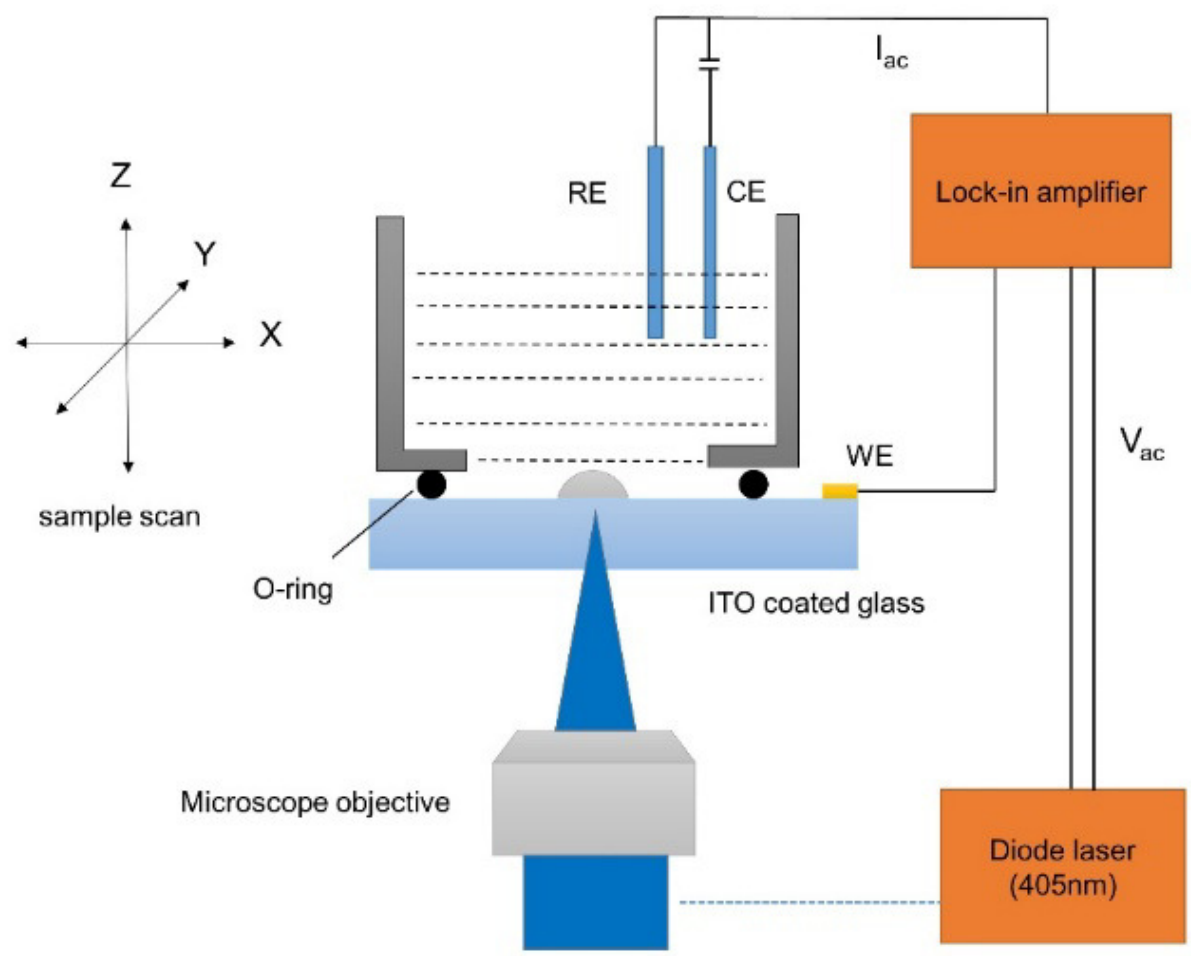

Figure 1. Schematic of the LAPS setup by scanning a focused, modulated laser beam across ITO coated glass.

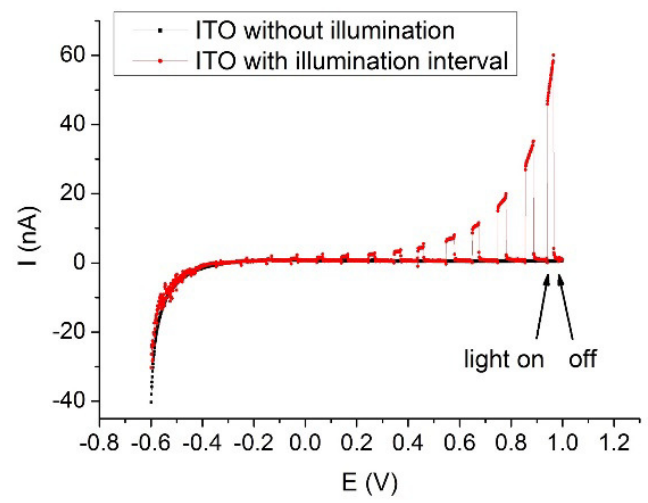

Figure 2. LSV curves of ITO coated glass in the dark and with chopped illumination. 
(A)

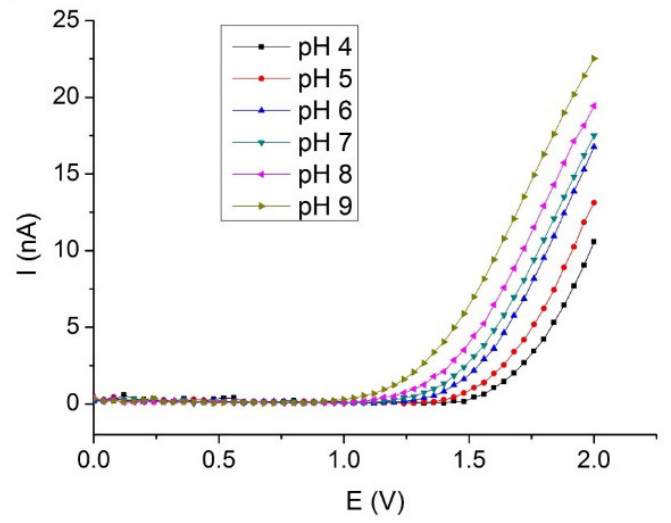

(B)

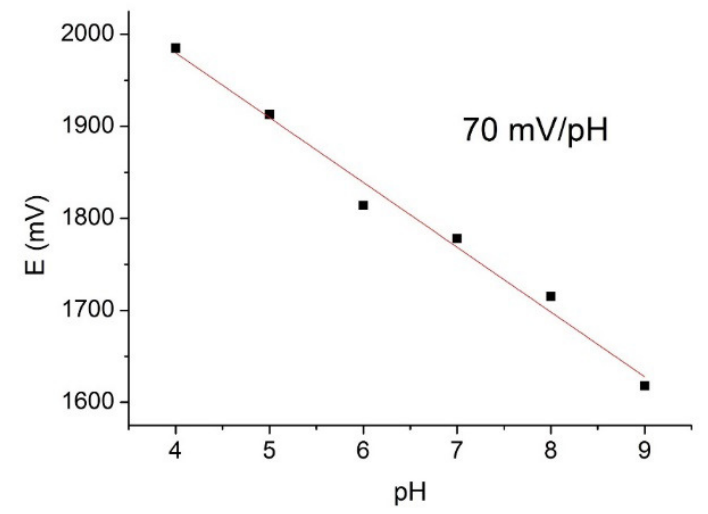

Figure 3. (A) LAPS I-V curves of ITO coated glass at different $p H$ values; (B) the $p H$ sensitivity of the ITO coated glass $\left(R^{2}=0.98\right)$. 
(A)

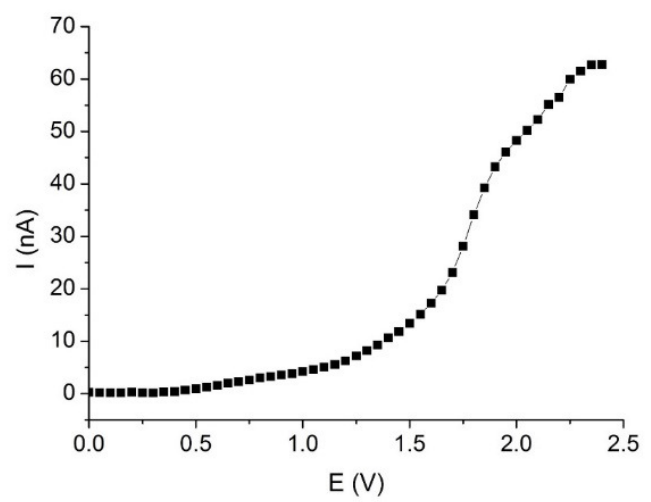

(B)

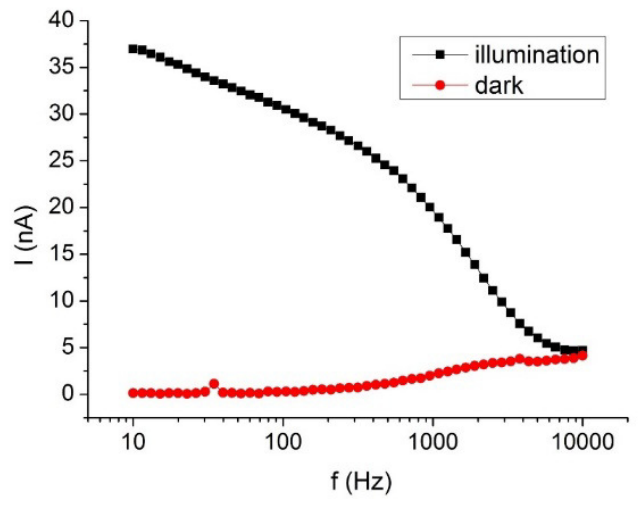

Figure 4. (A) LAPS I-V curve of blank ITO in pH 7.4 PBS buffer at $10 \mathrm{~Hz}$ with an unfocused laser source; (B) The frequency dependence of the ac photocurrent and background ac current in the dark. 
(A)

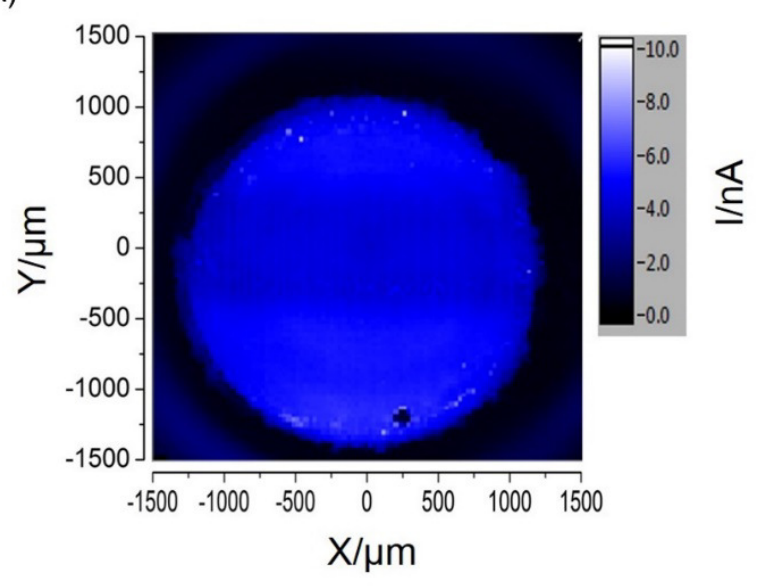

(B)

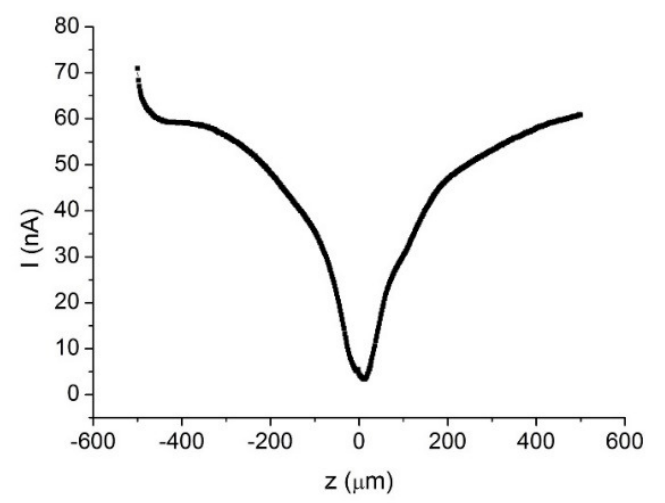

(C)

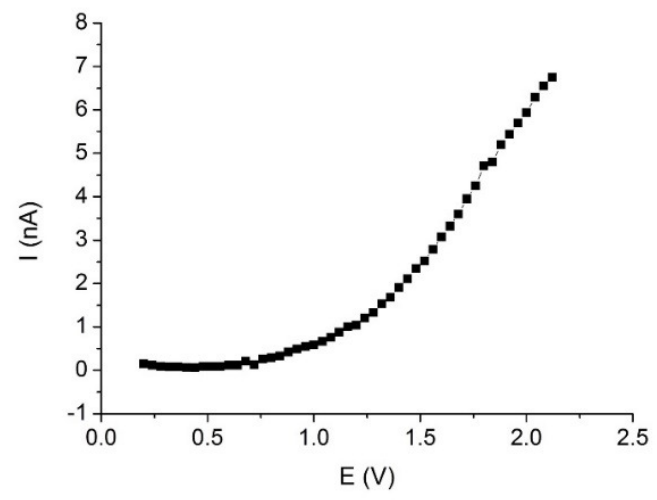

Figure 5. (A) The LAPS image of blank ITO at $2 \mathrm{~V}$ and $10 \mathrm{~Hz}$ with a focused laser beam; (B) $z$ axis line scan of the photocurrent around the focus; $(C)$ the LAPS I-V curve in focus at $10 \mathrm{~Hz}$. 
(A)

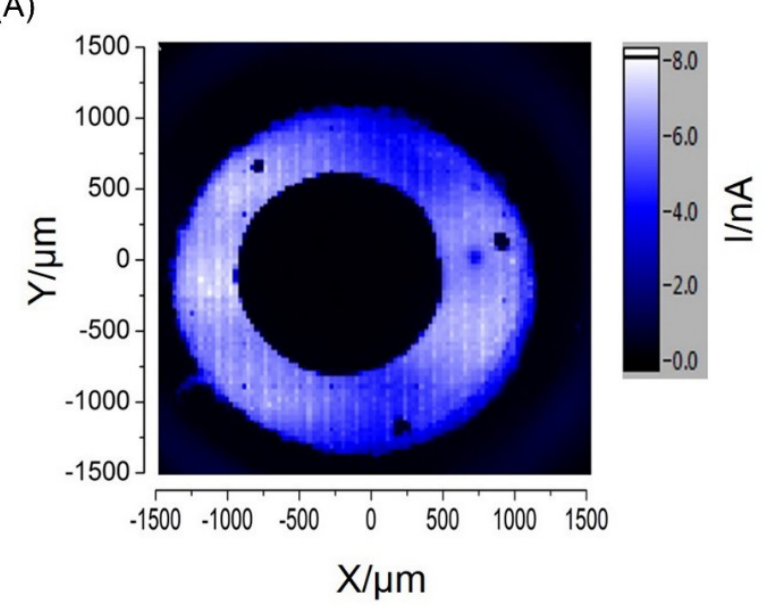

(B)

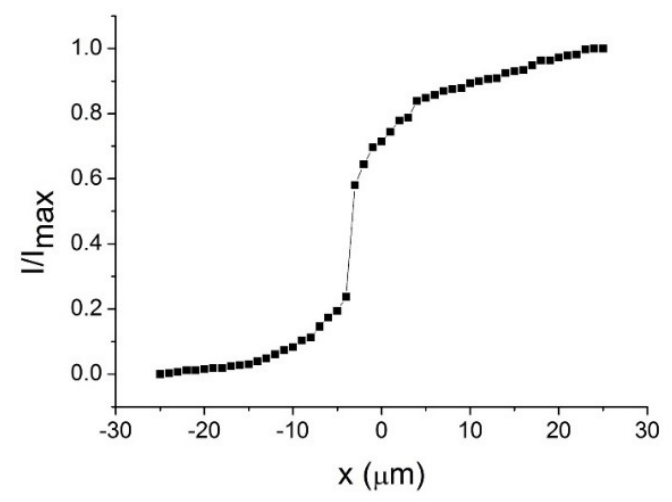

(C)

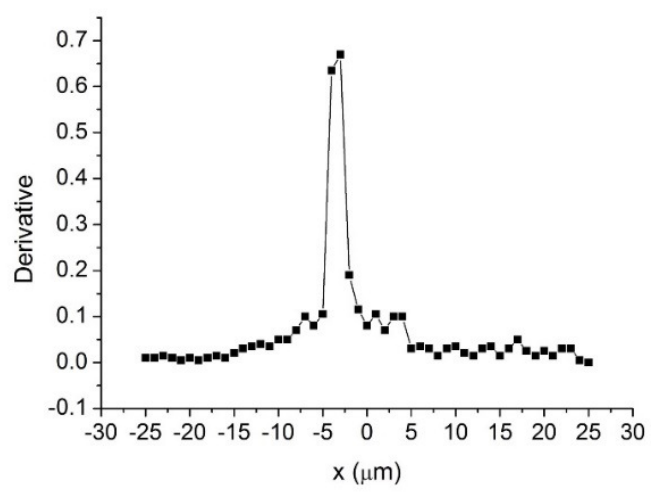

Figure 6. (A) The LAPS image of a PMMA dot at $2 \mathrm{~V}$ and $10 \mathrm{~Hz}$ with a focused laser beam; (B) $x$-axis photocurrent scan across the edge of the PMMA dot; $(C)$ the first derivative of $(B)$ with FWHM of 2.3 $\mu m$ 
For TOC only

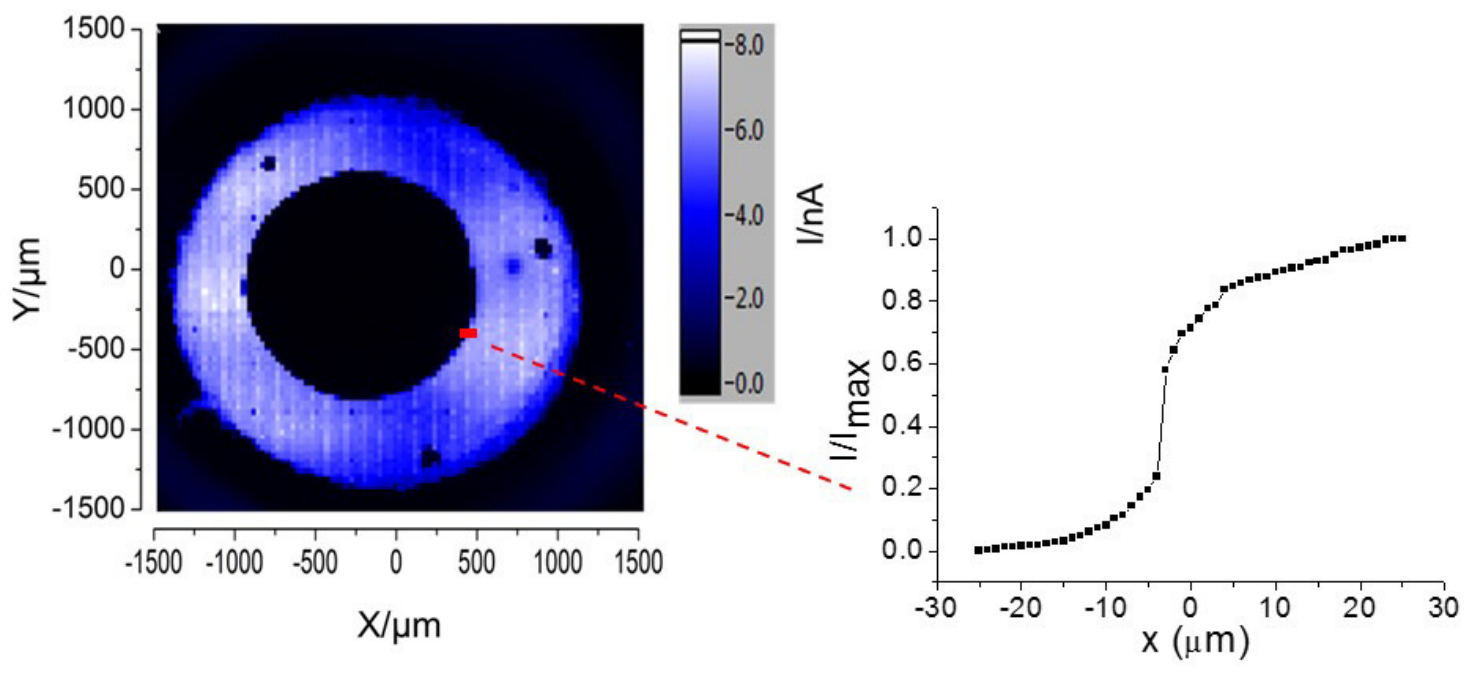

